\title{
Brain Atlas Deformation in the Presence of Large Space-Occupying Tumors
}

\author{
B. M. Dawant ${ }^{1}$, S. L. Hartmann ${ }^{2}$, and S. Gadamsetty ${ }^{1}$ \\ ${ }^{1}$ Department of Electrical and Computer Engineering \\ ${ }^{2}$ Department of Biomedical Engineering \\ Vanderbilt University, Nashville, Tennessee \\ \{dawant,slh,srikanth\}@vuse.vanderbilt.edu
}

\begin{abstract}
Brain atlases contain a wealth of information that could be used for radiation therapy or neurosurgical planning. So far, however, when large space occupying tumors and lesions drastically alter the shape of brain structures and substructures, atlas-based methods have been of limited use. In this work we present a new technique that permits warping a brain atlas onto image volumes in which large lesions are present. This technique involves several steps: a global registration to bring the two volumes into approximate correspondence, a local registration to warp the atlas onto the patient volume, the seeding of the warped atlas with a synthetic tumor, and the deformation of the seeded atlas. Global registration is performed using a mutual information criterion. The method we have used for atlas warping is derived from optical flow principles. Preliminary results obtained on real patient images are being presented. These results indicate that the method we propose can be used to automatically segment structures of interest in brains with gross deformation. Potential areas of application for this method include automatic labeling of critical structures for radiation therapy and presurgical planning.
\end{abstract}

Keywords: brain atlas, registration, deformation, segmentation

\section{Introduction}

In recent years, atlas-based methods have been proposed to segment automatically structures and substructures in medical images. These methods rely on the existence of a reference image volume (the atlas) in which structures of interest have been carefully segmented, possibly by hand. To segment a new image volume, a transformation that registers the atlas to this volume is first computed. This transformation is then used to project labeled structures from the atlas onto the image volume to be segmented. Medical image registration is a topic that has been studied by many researchers in the field over the years (see for instance Maintz [1] for a recent review article). But, the vast majority of these methods were developed to register multi-modal information (e.g. PET, CT, and MR) pertaining to a single subject. For this type of application, global transformation matrices involving 
rotation, translation, scaling, and possibly skew are sufficient. But, this type of transformation is unable to take into account non-linear differences between brain morphology and is thus inadequate for accurate atlas-based segmentation and labeling. A number of methods have been proposed that permit the computation of transformations with more degrees of freedom capable of warping one brain onto the other. Collins [2] has used a multi-resolution approach in which the overall non-linear transformation is composed of a set of local linear deformations obtained by maximizing the correlation of intensity and gradient features in the images to be registered. Bajcsy [3], [4] used an elastic model approach. Algorithms based on viscous fluid models were put forth by Christensen [5] and Bro-Nielsen [6]. Meyer $^{\circ}[7]$ has proposed a technique based on thin-plate splines in which an optimizer is used to adjust the position of homologous control points in homologous data sets. Rueckert [8] uses a similar approach but with B-splines. Woods [9] relies on polynomials up to order five. Thirion [10] proposed a method called "demons" that is similar to an optical flow approach for small displacements and that trades the rigor of physical modeling for simplicity of implementation and speed of execution. These techniques have great potential for the creation of statistical atlases or for the comparison of morphological characteristics between populations. But, these methods have been limited to cases without gross anatomical abnormalities. Atlas-based methods have therefore not yet been used for applications such as radiation therapy or presurgical planning when tumors dramatically alter the morphology of the brain. In a recent paper [11], a method based on a biomechanical model of the brain has been proposed for atlas deformation in the presence of gross abnormalities. The proposed method consists of several steps. First, the tumor is shrunk to a small mass, resulting in an estimate of the brain before tumor growth. Tumor shrinking is modeled as a uniform contraction and brain deformation resulting from this contraction is computed using finite element methods. This results in a "normal" brain that can be registered to an atlas. After registration, the tumor is grown back to its original shape using the inverse of the procedure used for shrinking the tumor. We have the same goal as Kyriacou and Davatzikos, i.e., we want to be able to use the wealth of information provided by atlases for applications such as radiation therapy or surgical planning even when large tumors drastically alter brain morphology, but our approach is different. In particular, we do not rely on a biomechanical model of the brain and we do not need to shrink the tumor to be able to register the normal atlas with the pathological brain. In the remainder of this paper we describe our method, present results we have obtained on real patient data, and discuss ways by which our current method can be improved.

\section{Methods}

\subsection{Data Sets}

Results presented in this study were obtained on two data sets acquired with a General Electric 1.5 Tesla Signa MR scanner using a spoiled gradient echo pulse sequence. Each volume consists of 128 sagittal slices, and each slice has dimensions of $^{\circ} 256^{\circ} \mathrm{x}^{\circ} 256$ pixels. Voxel dimensions are $.94 \times .94 \times 1.3 \mathrm{~mm}^{3}$. Both the volumes have been obtained after injection of Gadolinium. 


\subsection{Atlas Deformation}

Our method involves the following steps. First, a similarity transformation (three rotation angles, three translation vectors, and three scaling factors) is computed to globally register the atlas and the patient volumes. The global transformation is computed using the MIRIT package developed at the Catholic University of Leuven ${ }^{\circ}[12]$. Mutual information is used as the similarity criterion. Next, a highly non-linear transformation that brings the atlas and the patient volume in local correspondence is computed. The warped atlas is then "seeded" with a small structure centered approximately on the centroid of the tumor. Finally, the non-linear deformation algorithm is applied to the seeded warped atlas. In this step, the seeded tumor is grown until it reaches the size of the actual tumor and surrounding tissues in the atlas are displaced and deformed.

Local transformation: Thirion has presented the problem of image matching in terms of "demons" (by analogy with Maxwell's demons). This is a general framework in which object boundaries in one image are viewed as semi-permeable membranes. The other image, considered as a deformable grid, diffuses through these interfaces driven by the action of effectors (the demons) situated within the membranes. Various kinds of demons can be designed to apply this paradigm to specific applications. In the particular case of deformations based on voxel-by-voxel intensity similarity, the demons paradigm is similar to optical flow methods. It is an independent implementation of this approach [13] in which the displacememt vector for each voxel is computed as follows that has been used in this study.

$$
\vec{v}_{I_{2} \rightarrow I_{1}}=\frac{\left(I_{2}-I_{1}\right) \vec{\nabla} I_{1}}{\vec{\nabla} I_{1}^{2}+\left(I_{2}-I_{1}\right)^{2}}
$$

With I1 and I2 the intensity values of he images to be matched. In this approach, global smoothness of the displacement field is not enforced. Rather than using a global regularization method, a more local constraint imposing similar displacements for nearby pixels can be imposed by smoothing this field with, for instance, a Gaussian filter. The standard deviation of the Gaussian filter can also be used to change the characteristics of the matching transformation. The larger the standard deviation of the filter, the less elastic the transformation.

It should also be mentioned that large morphological differences between image volumes could render optical flow methods completely ineffective because the assumption of small displacement is violated. Two mechanisms have been used to make the algorithm more robust to large differences. First, the algorithm is applied in a hierarchical way. Second, a mechanism has been implemented that maintains compatibility between the forward and the reverse deformation fields. As proposed in $^{\circ}[10],[15]$ this is done by computing the deformation field T12 (the deformation field warping image 1 onto image 2 ) and the deformation field T21 (the deformation field warping image 2 onto image 1 ) and distributing the residual $\mathrm{R}=\mathrm{T} 12^{\circ} \mathrm{T} 21$ onto these two fields. This construct, coupled with the smoothing of the field prevents its 
tearing as well as crossing over of neighborhood pixels. It also provides a way to obtain the inverse transformation.

Atlas seeding. As the results will show, after the first two steps (i.e, similarity transformation and atlas deformation), the atlas and the brain to segment are in correspondence except in regions that have been drastically deformed by the tumor. The tumor is then outlined manually in the patient volume and the inside of this contour is assigned an intensity value different from the surrounding tissues. The seed is created by eroding the tumor mask of the patient, and is placed into the atlas volume.

Seeded atlas deformation. Once the atlas volume has been seeded, the deformation algorithm described earlier is applied a second time. This time, the algorithm attempts to warp the seeded atlas onto the patient image. Because the atlas has been seeded with a small region with the same intensity value as the delineated tumor, the algorithm grows the tumor seed until it reaches the size of the tumor in the patient volume. Because the deformation field is regularized with a Gaussian filter, the displacement field generated by the growth of the tumor also displaces surrounding tissues. As discussed earlier, the size and the standard deviation of the Gaussian filter used to regularize the displacement field determine the characteristics of the warping transformation. A small standard deviation only imposes very local constraints, thus resulting in transformations that are more elastic than those obtained with larger standard deviations. Here we have used two values for the standards deviation of the Gaussian filter. One for the 3D warping of the original atlas to the patient volume (step two in our approach) and one for the 3D deformation of the seeded atlas. To warp the original atlas to the patient volume we have selected a relatively high sigma value (2.0). This value was obtained experimentally and it was found to be a good compromise between deforming the atlas and preserving its structural integrity. For larger values, the algorithm is unable to deform the atlas enough to register it accurately with the patient volume. Smaller values can lead to transformations that are completely inaccurate over areas affected by the tumor. Indeed, prior to seeding, the normal volume does not have any structure that corresponds to and could be warped onto the tumor. The algorithm could thus warp arbitrary structures in the atlas volume in an attempt to minimize intensity differences between the atlas and the patient volume. After seeding, the atlas and the patient volume have corresponding structures. The standard deviation of the smoothing filter can thus be reduced, which permits larger local deformation required to grow the tumor and displace surrounding tissues. For the data set used in this study a sigma of 0.5 was used for the 3D deformation of the seeded atlas. Again, this value was obtained experimentally.

\section{Results}

Figure 1 illustrates the results obtained after the first two steps. In each of these panels, the images on the right show one slice in a patient volume. The images on the left show the slice with the same index in the volume obtained after registering the atlas to the patient using a similarity transformation. The middle panels show the slice with the same index in the volume obtained by warping the volume on the left onto 
the volume on the right. The three top panels show one slice in the brain hemisphere without tumor; the three bottom panels show one slice in the hemisphere with a large space-occupying tumor.
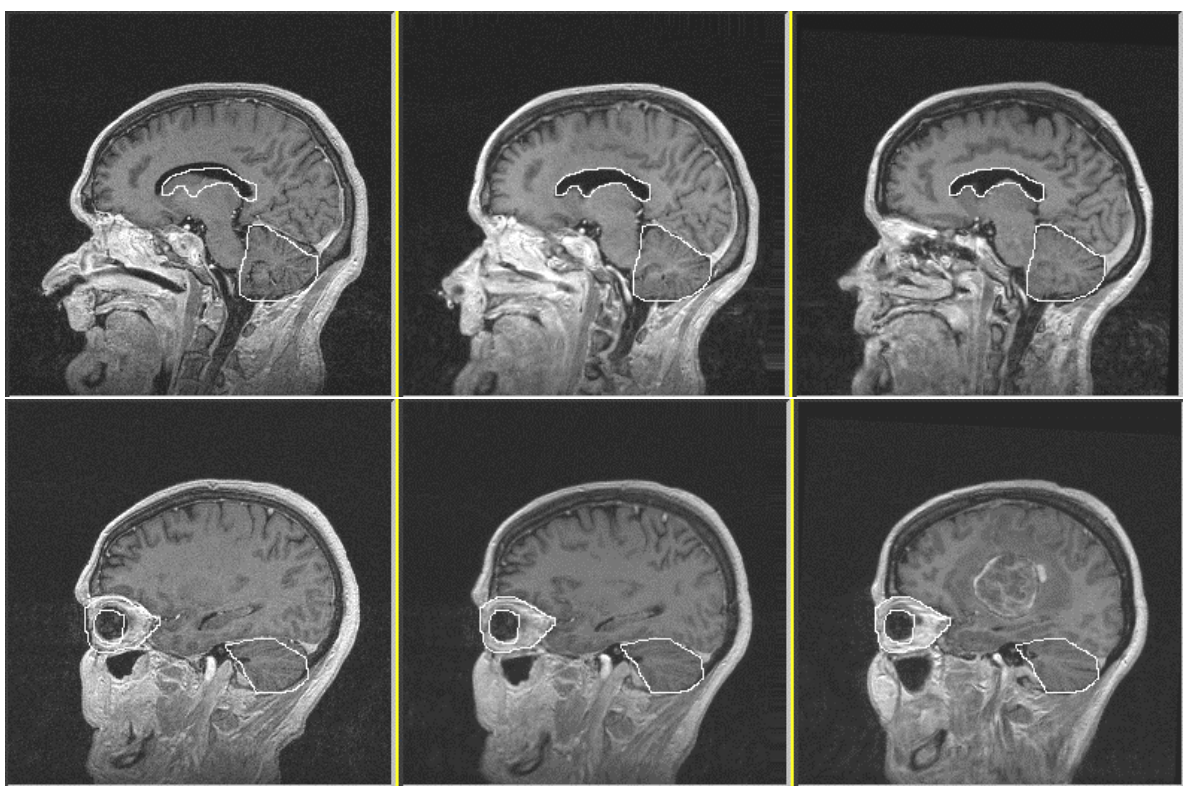

Fig. 1. Warping of the atlas to a patient volume. Right panels, patient volume. Left panels, global registration of the atlas to the patient volume. Middle panels, warping of the left volume onto the right volume. Top panels, hemisphere without tumor. Bottom panels, hemisphere with a large space-occupying tumor

Several important observations can be made from this figure. First of all, a nine degrees of freedom transformation is not sufficient to register the atlas to the patient volume. Contours have been drawn on the patient image (right panels) and echoed on the others. Note the registration inaccuracy around the ventricles, the cerebellum, and the eyes on the left panels. If a nine degrees of freedom transformation was sufficient, the contours drawn would also encircle precisely the regions of interest on the left panel images. Second, after warping (middle panels), structures that are far away from the tumor have been registered accurately, at least visually. A complete validation of this approach with quantitative validation on a number of structures of interest such as the optic nerve, optic chiasm, mamillary bodies, etc. has not yet been performed. Equally important is the fact that the integrity of the structures in the atlas has not been compromised by the presence of the tumor in the patient volume. Figure 1 clearly shows that over the tumor region the atlas has simply not been deformed to make it similar to the volume with a tumor. This is a highly desirable behavior. Indeed, when a tumor is present in the patient volume, there is no similar structure in the atlas to which it could be matched. The algorithm could thus attempt to deform an arbitrary structure and match it to the tumor, thus resulting in a completely erroneous warping of the atlas. 


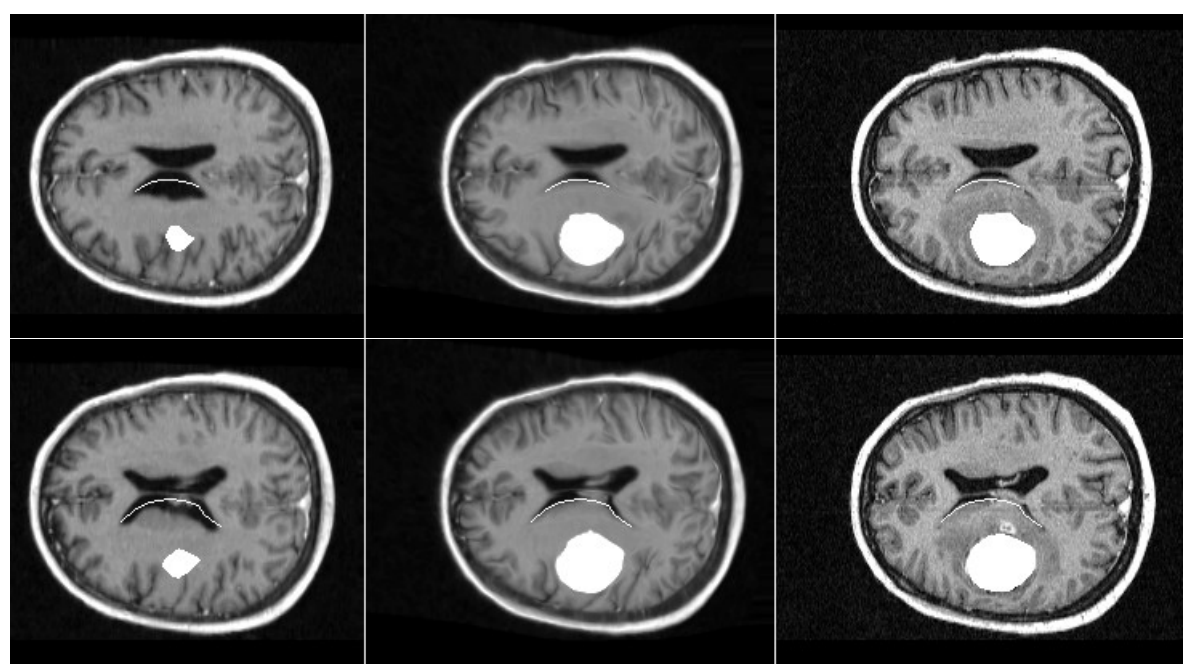

Fig. 2. Atlas seeding and deformation processes. Right, patient volume with manually delineated tumor. Left, warped atlas (same volume as left panel in figure 2) with tumor seed. Middle, seeded atlas after deformation. The top and bottom panels show different slice indexes.

Figure 2 illustrates the results obtained by seeding the atlas and warping the seeded atlas. The top and bottom panels of this figure show results on two different slices. The left panels in this figure are transverse slices in the volume obtained after deformation of the unseeded atlas. The right panels are transverse slices with the same index in the patient volume. The contour of the tumor has been delineated in the patient volume and the tumor has been assigned an arbitrary high value. The tumor region identified in the patient volume has been eroded to create the seed that has been placed in the warped atlas. The middle panels show the slices with the same indices in the volume obtained after warping the seeded atlas. Again, contours have been drawn on the patient volume (right panels) and copied on the other images. Prior to seed growing, the ventricles in the atlas kept their original shape. After seed growing, the ventricles and surrounding tissues such as the caudate have been displaced by the tumor. There is a good visual agreement between these contours and the actual structure boundary.

\section{Discussion}

This work is one of the first attempts at atlas deformation when the target volume contains large space-occupying lesions that drastically deform and displace normal anatomy. As opposed to our method, the approach proposed previously by Kyriacou and Davatzikos relies on biomechanical models. Their technique is based on a certain number of assumptions. First, white matter, gray matter, and cerebrospinal fluid regions should be known because the material constant in their model is different for each of these classes. Automatic segmentation of pathological image volumes may be 
challenging because the tumor and its edema may have intensity values similar to other tissue classes. More importantly, the tumor contraction algorithm used in their approach assumes the size of structures such as the ventricles prior to tumor growth to be known. Methods based on a-priori statistical information about ventricular shape and size have been proposed to address this problem but their effectiveness and accuracy has not yet been studied.

The method we propose does not require segmentation (except for the contours of the tumor in the patient volumes) and is not based on any explicit underlying mathematical model. This is both its strength and its potential weakness. It is its strength because the method is computationally simple and fast. The consistency of the deformation field is controlled by the smoothing filter and we have shown that by choosing values appropriately we can modify the behavior of the algorithm to obtain transformations that lead to accurate deformations both over normal and abnormal regions. The implicit assumption on which our approach is based is the local coherence of the deformation field, i.e., neighbor pixels are constrained to move in a similar way. The size of the smoothing filter defines the size of the neighborhood. The lack of an explicit underlying mathematical model is also the potential weakness of this approach. If the smoothing filter is not chosen correctly, the resulting transformation can be catastrophically wrong. It should also be noted, however, that, in our experience, both with normal volunteers [16] and with chronic alcoholics suffering from severe atrophy [17], the algorithm is robust with respect to the choice of the smoothing parameter.

Future work will involve improving the deformation algorithm by modeling structures of interest in the atlas volume. This will permit the automatic adaptation of the smoothing parameters according to the physical properties of these structures as well as guaranteeing the physical plausibility of the deformation field. Structures such as the optic nerve, optic chiasm, mamillary bodies, spinal cord, putamen, globus pallidus, etc. can indeed be labeled in the atlas and represented as 3D objects. The consistency of the deformation field could thus be imposed over these objects rather than on arbitrary neighborhoods, thus guaranteeing structural integrity in the deformed images.

\section{Acknowledgements:}

This work was supported in part by NIH grant R01 AA 10583.

\section{References}

1. J.B. Maintz and M.A. Viergever, A survey of medical image registration. Medical Image Analysis, vol. 2, no. 1, Apr, 1998.

2. D.L. Collins, P. Neelin, T.M. Peters, and A.C. Evans, Automatic 3D intersubject registration of MR volumetric data in standardized Talairach space. Journal of Computer Assisted Tomography, vol. 18, no. 2, pp. 192-205, 1994.

3. R. Bajcsy and S. Kovacic, Multiresolution elastic matching. Computer Vision, Graphics, and Image Processing, vol. 46, pp. 1-21, 1989. 
4. J.C. Gee, M. Reivich, and R. Bajcsy, Elastically deforming 3D atlas to match anatomical brain images. Journal of Computer Assisted Tomography, vol. 17 , no. 2, pp. 225-236, 1993.

5. G.E. Christensen, M.I. Miller, and M. Vannier, 3D brain mapping using a deformable neuroanatomy. Phys Med Biol, vol. 39, pp. 609-618, 1994.

6. M. Bro-Nielsen and C. Gramkow, Fast fluid registration of medical images. eds. K.H. Hohne and R. Kikinis. 1131, pp. 267-276, 1996. Visualization in Biomedical Computing. Hamburg, Germany.

7. C.R. Meyer, J.L. Boes, B. Kim, P. Bland, K.R. Zasadny, P.V. Kison, K. Koral, K.A. Frey, and R.L. Wahl, Demonstration of accuracy and clinical versatility of mutual information for automatic multimodality image fusion using affine and thin plate spline warped geometric deformations. Medical Image Analysis, vol. 3, pp. 195-206, 1997.

8. D. Rueckert, C. Hayes, C. Studholme, P. Summers, M. Leach, and D.J. Hawkes, Non-rigid registration of breast MR images using mutual information. MICCAI Proceedings, 1496, pp. 1144-1152, 1998.

9. R.P. Woods, S.T. Grafton, J.D.G. Watson, N.L. Sicotte, and J.C. Mazziotta, Automated image registration: II. Intersubject validation of linear and nonlinear models. Journal of Computer Assisted Tomography, vol. 22, no. 1, pp. 153-165, 1998.

10.J.-P. Thirion, Image matching as a diffusion process: an analogy with Maxwell's demons. Medical Image Analysis, vol. 2, no. 3, pp. 243-260, 1998.

11.S.K. Kyriacou and C. Davatzikos, A biomechanical model of soft tissue deformation, with applications to non-rigid registration of brain images with tumor pathology. MICCAI Proceedings, pp. 531-538, 1998.

12.F. Maes, A. Collignon, D. Vandermeulen, G. Marchal, and P. Suetens, Multi-modality image registration using information theory, MIRIT users manual, Version 97/08. Internal report, Catholic University of Leuven, 1997.

13.S.L. Hartmann, Automatic segmentation of medical images using optical flow based atlas deformation. Master's Thesis, Department of Biomedical Engineering, Vanderbilt University, 1998.

14.B. Horn and B. Schunck, Determining optical flow. Artificial Intelligence, vol. 17, pp. 185203, 1981.

15.D.J. Burr, A dynamic model for image registration. Computer Graphics and Image Processing, vol. 15, pp. 102-112, 1981.

16.B.M. Dawant, J.-P. Thirion, F. Maes, D. Vandermeulen, and P. Demaerel, Automatic 3D segmentation of internal structures of the head in MR images using a combination of similarity and free form transformations. SPIE Medical Imaging, 1998.

17.S.L. Hartmann, M.H. Parks, H. Schlack, W.R. Riddle, R.R. Price, P.R. Martin, and B.M. Dawant, Automatic Computation of Brain and Cerebellum Volumes in Normal Subjects and Chronic Alcoholics. IPMI Proceedings, In Press, 1999. 